\title{
Paraclinoid Carotid Artery Aneurysms with the Dome Eroding the Anterior Clinoid Process: Preoperative Depiction of Topography and Surgical Tactics
}

\author{
- Two Case Reports-
}

\author{
Shiro NAGASAWA, Ryuhsuke OGAWA, Yoshio SuYAMA, and Tomio OHTA \\ Department of Neurosurgery, Osaka Medical College, Takatsuki, Osaka
}

\begin{abstract}
A 70-year-old male and a 55-year-old female presented with unruptured aneurysms in the clinoid segment of the carotid artery. Intraoperative rupture during removal of the anterior clinoid process resulted in unintended carotid artery occlusion in the former patient, causing hemispherical infarction and death. However, the aneurysm was successfully clipped due to preoperative recognition of the aneurysmal dome in the clinoid process in the latter. Magnetic resonance angiography and three-dimensional computed tomography angiography source images provide detailed visualization of the topographic anatomy around the paraclinoid aneurysms and are helpful for definite surgical planning.
\end{abstract}

Key words: magnetic resonance angiography, cerebral aneurysm, ophthalmic artery aneurysm, paraclinoid aneurysm, three-dimensional computed tomography angiography

\section{Introduction}

Aneurysms originating from the clinoid portion of the internal carotid artery are surrounded by a number of structures within a narrow space at the skull base, and are variously called ophthalmic, clinoid, paraclinoid, carotid cave, $\mathrm{C}_{2}, \mathrm{C}_{2}-\mathrm{C}_{3}$, or $\mathrm{C}_{3}$ aneurysms. ${ }^{1-4,7,9,12)}$ These aneurysms carry a risk of subarachnoid hemorrhage unless completely confined within the cavernous sinus. These aneurysms may erode the anterior clinoid process, so removal of this process in the usual way would result in unexpected aneurysmal rupture. ${ }^{8)}$ Therefore, careful evaluation of the topography around the aneurysm is essential for surgical planning and treatment. However, the anterior clinoid process and internal carotid artery show significant variations in size and position, and are located in such a narrow space that neither conventional angiography nor computed tomography (CT) can always provide sufficient information.

Magnetic resonance (MR) angiography and three-

Received December 7, 1995; Accepted April 5, 1996 dimensional (3-D) CT angiography have been widely used in the diagnosis of cerebral aneurysms, ${ }^{6,11)}$ but the usefulness of their source images for aneurysmal surgery is less well known. ${ }^{10)}$ Since these images are essentially a series of thin slices in the base view, the skull base structures are visualized without overlap.

We describe two cases of paraclinoid carotid artery aneurysms in which the axial source images of MR or 3-D CT angiography were of great value in understanding the anatomical relationship of the aneurysm to the carotid artery, optic nerve, and the anterior clinoid process.

\section{Case Reports}

Case 1: A 70-year-old male presented with transient ischemic attack. MR angiography (1.5 Tesla; Signa Advantage; GE Medical Systems, Waukesha, Wis., U.S.A.) incidentally suggested the presence of an aneurysm (Fig. 1). Conventional angiography demonstrated an aneurysm originating from the $\mathrm{C}_{3}$ segment of the right internal carotid artery and extending superolaterally (Fig. 2). No other disorder was observed in the cerebral arteries or tissues.

Standard right pterional craniotomy was per- 


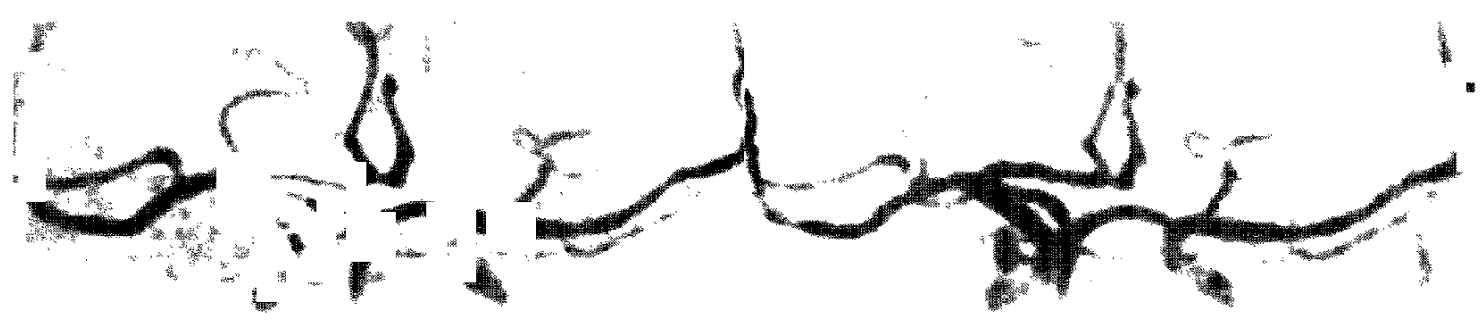

Fig. 1 Case 1. MR angiography stereoscopic projection images depicting an aneurysm at the $\mathrm{C}_{3}$ segment of the right carotid artery.

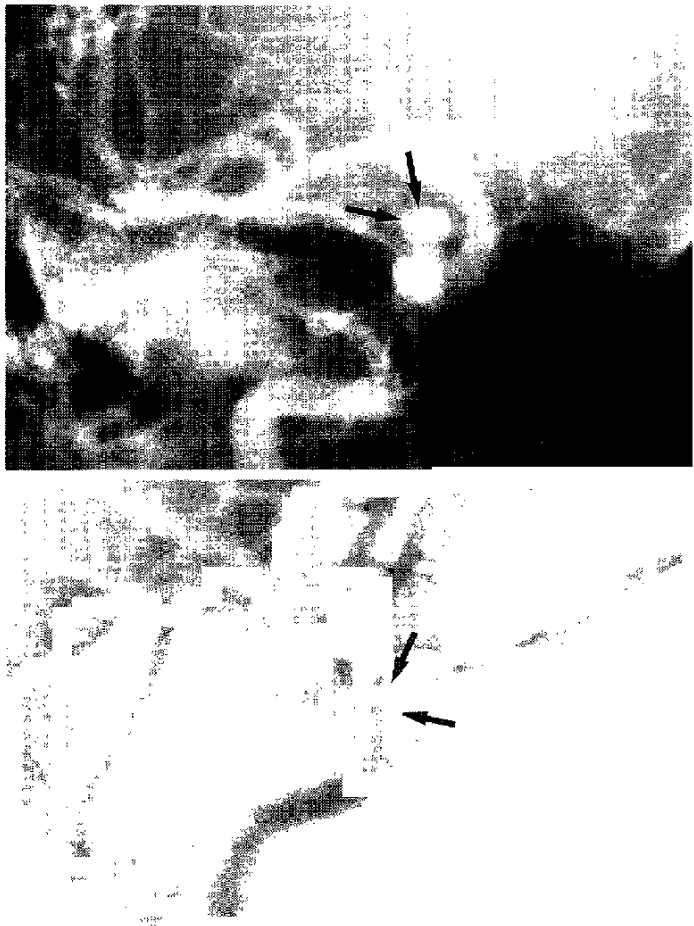

Fig. 2 Case 1. Right carotid angiograms, anteroposterior (upper) and lateral (lower) views, demonstrating an aneurysm $8 \mathrm{~mm}$ in diameter (arrows) originating from the $\mathrm{C}_{3}$ segment of the carotid artery and extending superiorly.

formed, but neither an aneurysm nor a bulge was found intradurally near the anterior clinoid process. Some drilling of the clinoid process after removal of the dura was followed by massive arterial bleeding from within. Immediate exposure and temporary occlusion of the cervical carotid artery reduced the bleeding. Further removal of the clinoid confirmed the presence of the aneurysmal neck at the $C_{3}$ segment of the artery, but the neck was too fragile for clipping due to atherosclerotic changes. The carotid artery was trapped between the cervical and $\mathrm{C}_{2}-\mathrm{C}_{3}$ junctions. Since the tolerance test for carotid artery occlusion had not been carried out before surgery, a saphenous vein graft was placed between the common carotid artery and the $\mathbf{M}_{2}$ portion of the middle cerebral artery.

Although arterial flow was restored within 6 hours of occlusion and the brain showed no obvious abnormalities at closure, postoperative CT scan revealed infarction of the entire right hemisphere. He died 7 days later.

The preoperative craniograms, conventional angiograms, and CT scans were reviewed, but none demonstrated erosion or round defect of the clinoid process. The axial source images of MR angiography provided an accurate representation of the topography around the aneurysm, showing the dome originating from the lateral surface of the $C_{3}$ segment of the carotid artery, extending superiorly and invading the inferior portion of the clinoid process (Fig. 3). Case 2: A 55-year-old female presented with visual field defect due to pituitary adenoma. MR angiography (1.5 Tesla; MRH-1500; Hitachi, Tokyo) and 3-D CT angiography found an aneurysm incidentally. Digital subtraction angiography demonstrated the aneurysm originating from the $\mathrm{C}_{2}$ or $\mathrm{C}_{3}$ segment of the right carotid artery and extending superolaterally (Fig. 4). The axial source images of MR angiography demonstrated that the aneurysm arose from the lateral surface of the $C_{2}-C_{3}$ junction of the carotid artery and extended superiorly into the clinoid, with the optic nerve located anteromedial to the dome (Fig. 5). 3-D CT angiography showed a bulge of the carotid artery near the anterior clinoid process suggesting the presence of an aneurysm, but the dome in the extradural space was not identified. However, the axial source images clearly demonstrated that the aneurysmal dome had eroded the inferior portion of the clinoid process (Fig. 6).

The cervical carotid artery was exposed via the 


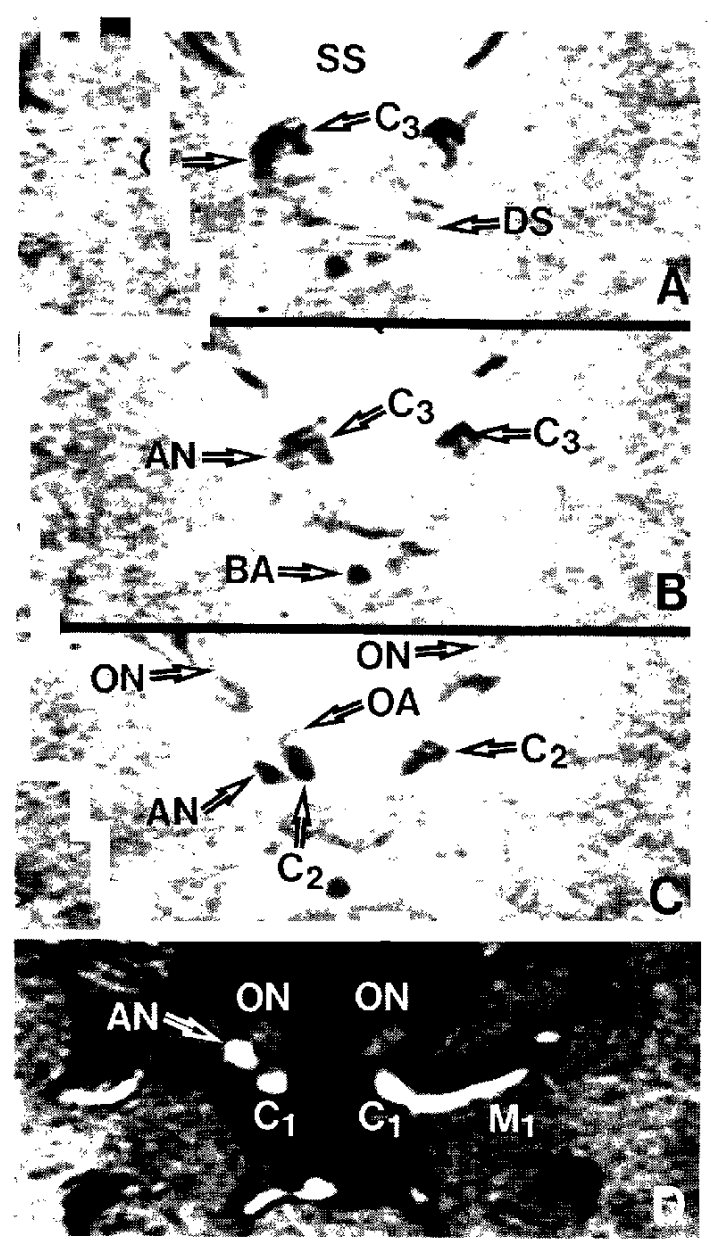

Fig. 3 Case 1. MR angiography axial source images, located $0.7 \mathrm{~mm}$ (B), $2.1 \mathrm{~mm}$ (C), and $7.0 \mathrm{~mm}$ (D) superior to $\mathrm{A}$, showing the aneurysm (AN) has developed at the lateral wall of the $C_{3}$ segment of the right carotid artery, contacting the medial surface of the anterior clinoid process, then extending into the clinoid process at the level of the exit of the ophthalmic artery (OA). The dome within the clinoid, the $\mathrm{C}_{1}$ segment, and the right optic nerve $(\mathrm{ON})$ are adjacent. BA: basilar artery, SS: sphenoid sinus, DS: dorsum sellae.

pterional approach on the right side. No aneurysm was observed around the anterior clinoid process. The pituitary adenoma had elevated the right optic nerve, so was removed first to decompress the nerve. Drilling was then initiated in the superolateral wall of the optic canal and continued to the anteromedial portion of the clinoid process, tracing the superomedial wall of the $\mathrm{C}_{2}$ segment proximally toward the $\mathrm{C}_{3}$ segment and the aneurysm. Minor bleeding was encountered on removal of small pieces

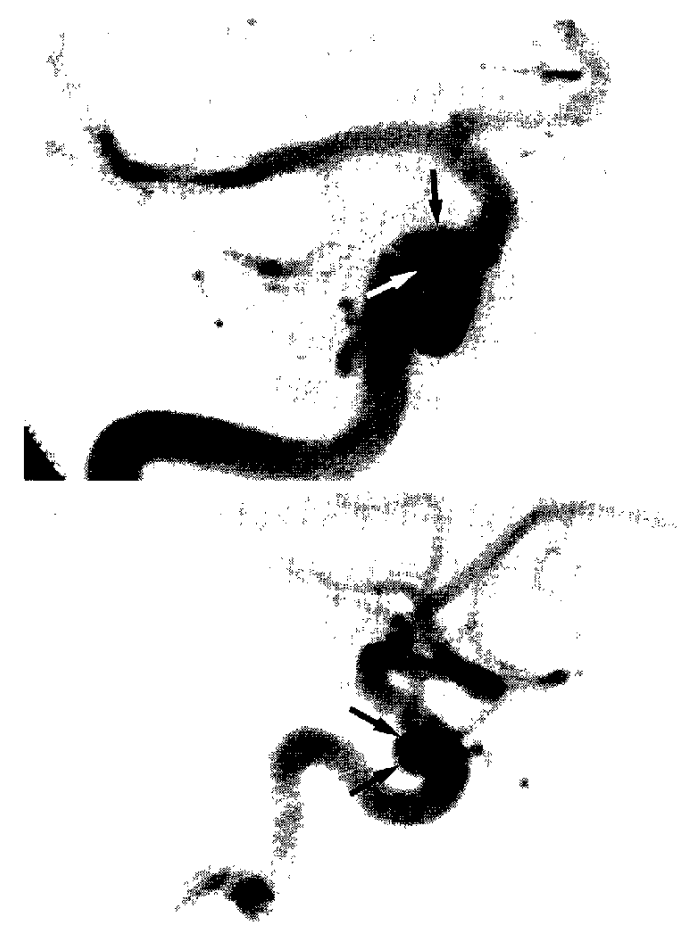

Fig. 4 Case 2. Right carotid angiograms, anteroposterior (upper) and lateral (lower) views, demonstrating an aneurysm $7 \mathrm{~mm}$ in diameter (arrows) originating from the lateral wall of the $\mathrm{C}_{2}$ or $\mathrm{C}_{3}$ segment of the carotid artery and extending superolaterally.

of the thin bony shell, but was well controlled. The aneurysm was found on the anterolateral wall of the $\mathrm{C}_{3}$ segment proximal to the dural ring, and was clipped successfully.

The postoperative course was uneventful.

\section{Discussion}

The aneurysms in our patients were first suggested by MR angiography and/or 3-D CT angiography, and then confirmed by conventional angiography. The aneurysms were classified as the subtype termed transitional cavernous aneurysm ${ }^{1)}$ or proximal posterior carotid artery wall aneurysm, ${ }^{2)}$ and were both very similar to the "subclinoid" aneurysm type. ${ }^{8}$ Unexpected aneurysmal rupture occurred during the initial step of removing the clinoid process in our first patient, suggesting that great care should be taken not to rupture the aneurysm during removal of the anterior clinoid process when the dome extends superolaterally and the clinoid process is markedly eroded. In our second patient, the carotid artery was 

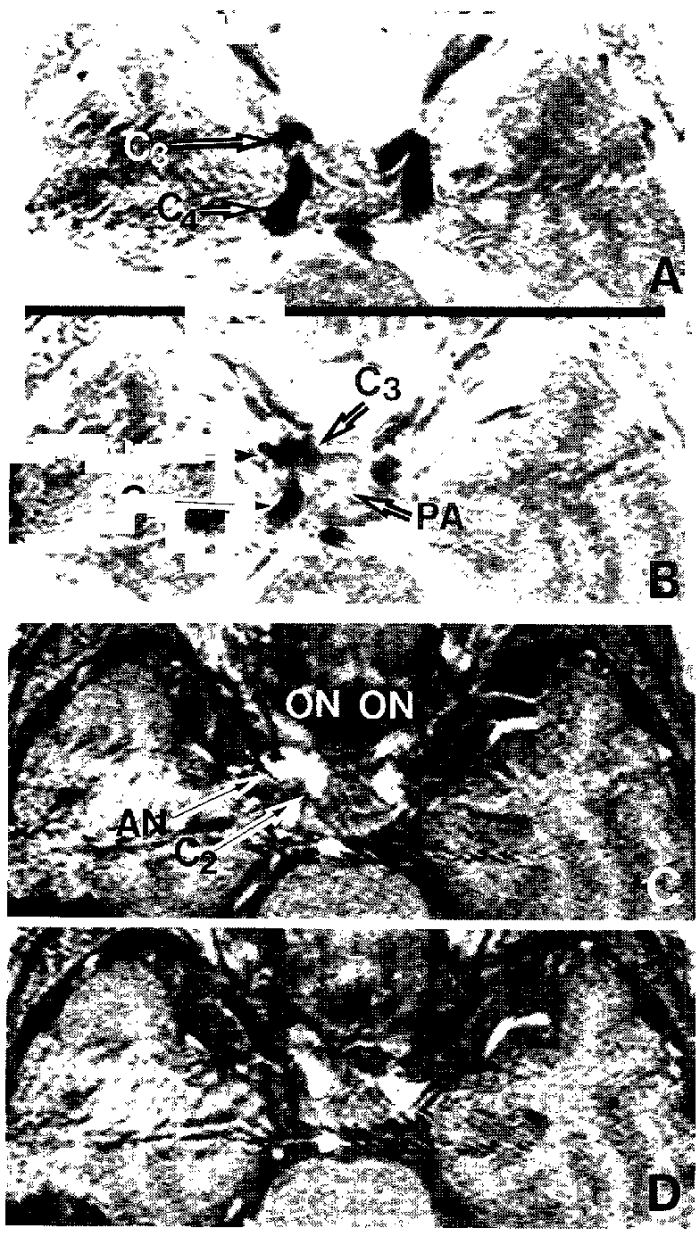

Fig. 5 Case 2. MR angiography axial source images, located $2.5 \mathrm{~mm}$ (B), $3.5 \mathrm{~mm}$ (C), and $4.5 \mathrm{~mm}$ (D) superior to $A$, showing an aneurysm at the lateral wall of the $C_{2}-C_{3}$ segment of the right carotid artery with extension superolaterally into the anterior clinoid process. The right optic nerve is adjacent to the $\mathrm{C}_{2}$ segment. PA: pituitary adenoma.

exposed at the neck and intradural removal of the anteromedial portion of the clinoid was performed to trace the superomedial wall of the $\mathrm{C}_{2}$ segment proximally toward the $\mathrm{C}_{3}$ segment and the aneurysmal neck.

Source images of MR angiography in this patient demonstrated the anatomical structures including each segment of the carotid artery, the neck and dome of the aneurysm, the optic nerve in both the optic canal and the subarachnoid space, and the ophthalmic artery. The anterior clinoid process could be traced as an area surrounded by a low intensity rim consistent with the cortical bone. Both MR and 3-D CT angiograms were obtained in the second

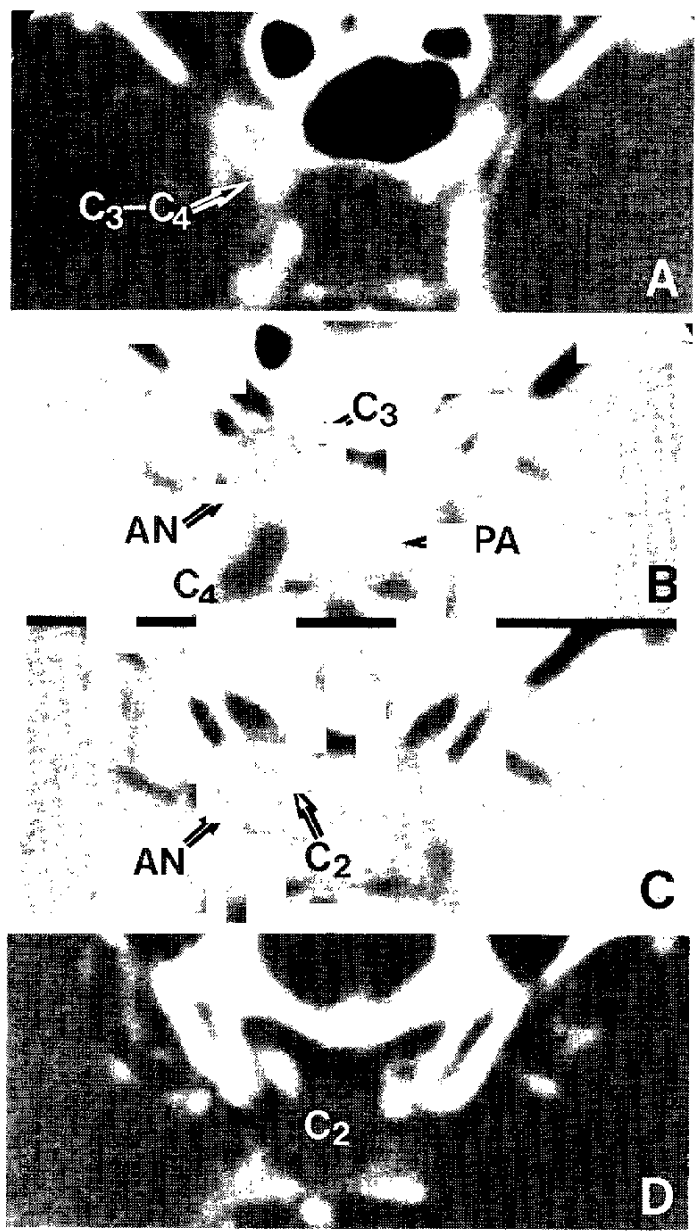

Fig. 6 Case 2. 3-D CT angiography axial source images, located $1.0 \mathrm{~mm}(\mathrm{~B}), 2.0 \mathrm{~mm}(\mathrm{C})$, and 3.0 $\mathrm{mm}$ (D) superior to $A$, showing an aneurysm originating from the posterolateral wall of the $\mathrm{C}_{2}-\mathrm{C}_{3}$ segment of the carotid artery, extending superiorly, and eroding the inferomedial portion of the clinoid process on the right.

patient. The source images of both visualized the carotid artery and the aneurysm, but MR source images more clearly demonstrated the optic nerve and 3-D CT source images showed the eroded clinoid process better.

An area in the clinoid process appearing as low density on CT scans or low intensity on MR images should be differentiated from pneumatization. ${ }^{5,8)}$ However, if MR or 3-D CT angiography shows an aneurysm within the eroded area in the clinoid process, further differentiation is not required.

Erosion of the clinoid may be suspected after careful evaluation of a paraclinoid aneurysm on conventional angiograms, but direct visualization of the 
eroded clinoid and the surrounding structures on 3-D CT or MR angiography source images is more helpful for definite surgical planning.

\section{Acknowledgments}

The authors wish to thank Dr. E. Tsuda, Department of Neurosurgery, Soseikai General Hospital, Kyoto for permission to publish the data on Case 1.

\section{References}

1) Al-Rodhan NRF, Piepgras DG, Sundt TM Jr: Transitional cavernous aneurysms of the internal carotid artery. Neurosurgery 33: 993-998, 1993

2) Batjer HH, Kopitnik TA, Giller CA, Samson DS: Surgery for paraclinoidal carotid artery aneurysms. $J$ Neurosurg 80: 650-658, 1994

3) Day AL: Aneurysms of the ophthalmic segment. A clinical and anatomical analysis. $J$ Neurosurg 72 : 677-691, 1990

4) Dolenc VV: A combined epi- and subdural direct approach to carotid-ophthalmic artery aneurysms. $J$ Neurosurg 62: 667-672, 1985

5) Gean AD, Pile-Spellman J, Heros RC: A pneumatized anterior clinoid mimicking an aneurysm on MR imaging. Report of two cases. $J$ Neurosurg 71: 128132, 1989

6) Harbaugh RE, Schlusselberg DS, Jeffery R, Hayden S, Cromwell LD, Pluta D: Three-dimensional computerized tomography angiography in the diagnosis of cerebrovascular disease. $J$ Neurosurg 76: 408-414, 1992

7) Kobayashi S, Kyoshima K, Gibo H, Hegde SA, Takemae T, Sugita K: Carotid cave aneurysms of the internal carotid artery. $J$ Neurosurg 70: 216-221, 1989

8) Korosue K, Heros RC: "Subclinoid" carotid aneurysm with erosion of the anterior clinoid process and fatal intraoperative rupture. Neurosurgery 31: 356-360, 1992

9) Linskey ME, Sekhar LN, Horton JA, Hirsch WL Jr, Yonas $\mathrm{H}$ : Aneurysms of the intracavernous carotid artery: A multidisciplinary approach to treatment. $J$ Neurosurg 75: 525-534, 1991

10) Nagasawa $S$, Deguchi J, Arai $M$, Tanaka $H$, Ohta $T$ : Usefulness of thin axial images of computerized tomography angiography for surgery on paraclinoid carotid artery aneurysms. No Shinkei Geka 23: 677684, 1995 (in Japanese)

11) Ross JS, Masaryk TJ, Modic MT, Ruggieri PM, Haacke EM, Selman WR: Intracranial aneurysms: Evaluation by MR angiography. AJNR $\mathrm{Am} J \mathrm{Neu}$ roradiol 11: 449-456, 1990

12) Tanaka $Y$, Kobayashi S, Kyoshima K, Sugita K: Mul- tiple clipping technique for large and giant internal carotid artery aneurysms and complications: Angiographic analysis. $J$ Neurosurg 80: 635-642, 1994

Address reprint requests to: S. Nagasawa, M.D., Department of Neurosurgery, Osaka Medical College, 2-7 Daigaku-cho, Takatsuki, Osaka 569, Japan.

\section{Commentary}

Drs. Nagasawa et al. have described two cases with superolateral wall aneurysm of the infraclinoid internal carotid artery. Those aneurysms were detected incidentally by MR and/or 3D CT angiogram. The authors suggested that detailed study of the source images of MR and CT angiogram could predict the relationship between the dome of an aneurysm and the anterior clinoid process. The technique applied in MR angiogram is a time-of-flight that is basically the gradient-echo sequences. Blood flow moving perpendicular to the scan plane appears bright on gradient-echo images. Although it is a sensitive tool for visualizing the arterial flow and cortical bone, it suffers from reduced $\mathrm{T}_{1}$ contrast, limited signal to noise ratio, and increased magnetic susceptibility artifacts. Since the MR imaging around the anterior clinoid process is often plagued by the CSF, vascular pulsating artifact, and magnetic susceptibility between the aerated sinus and cortical bone, one has to be careful to differentiate the cortical bone from the CSF and other artifacts. We agree with the authors' opinion that CT angiographic source images provide more accurate information regarding the relationship between the dome of an aneurysm and the anterior clinoid process. However, reformatted coronal or oblique sagittal CT images would be superior to axial images in delineating topographic anatomy of the paraclinoid region.

The authors are to be complimented for calling attention to careful evaluation of the topography by imaging studies that is essential for surgical planning and treatment for paraclinoid carotid artery aneurysms.

Kyu Chang LEE, M.D. and Dong Ik KIM, M.D.*

Departments of Neurosurgery and ${ }^{*}$ Radiology

Yonsei University College of Medicine Seoul, Korea 